\title{
Adjuvant chemotherapy with tegafur/uracil administration after transcatheter arterial chemoembolization for advanced hepatocellular carcinoma
}

\author{
HIROKI UEDA, HIROTO TANAKA, YOHEI KIDA, HIROKO FUKUCHI and MASAKAZU ICHINOSE
}

Third Department of Internal Medicine, Wakayama Medical University, Japan

Received December 21, 2007; Accepted February 4, 2008

\begin{abstract}
Although transcatheter arterial chemoembolization (TACE) is considered to be an effective treatment for advanced hepatocellular carcinoma (HCC), it is difficult to achieve complete necrosis by TACE alone due to incomplete embolization and tumor angiogenesis. Recent studies have shown that tegafur/uracil $\left(\mathrm{UFT}^{\circledR}\right)$ inhibits tumor angiogenesis in several cancer types. Therefore, this study was conducted to test the efficacy and toxicity of the UFT administration after TACE in advanced HCC. Thirty patients with HCC who had been treated with TACE alone more than three times and had a recurrence within 6 months were enrolled. All of the patients were treated with TACE and 28 patients were randomly assigned to the UFT (UFT $300 \mathrm{mg} /$ day, three days after TACE, $n=14)$ and control groups $(n=14)$. The primary end point was the time to treatment failure (TTF) and the secondary end points were mainly the response rate and toxicity. Administration and observation were continued up to 6 months after TACE unless local recurrence was detected or serious adverse events developed. The median TTF in the control group was 87 days, whereas in the UFT group it was 127 days, thus significantly prolonged as compared to the control group $(\mathrm{P}=0.0016)$. Moreover, the overall response rate $(35.7 \%)$ in the UFT group was significantly higher than that in the control group $(0 \%)$. As for toxicity, only 4 patients in the UFT group developed grade 1-2 toxicities such as ascites. Serious complications by TACE were not observed in either group. Notably, there were no increases in the serum VEGF levels in the UFT group whereas those in the control group increased significantly. In conclusion, UFT administration after TACE was an effective treatment and showed no severe
\end{abstract}

Correspondence to: Dr Masakazu Ichinose, The Third Department of Internal Medicine, Wakayama Medical University, 811-1 Kimiidera, Wakayama City 641-0012, Japan

E-mail: masakazu@wakayama-med.ac.jp hu11@wakayama-med.ac.jp

Key words: adjuvant chemotherapy, tegafur, uracil, transcatheter arterial chemoembolization, hepatocellular carcinoma adverse events. This regimen may have an adjuvant role and antiangiogenic function in advanced HCC.

\section{Introduction}

Hepatocellular carcinoma (HCC) is one of the most common cancers worldwide (1). Of the cases with $\mathrm{HCC},>80 \%$ are associated with liver cirrhosis. Transcatheter arterial chemoembolization (TACE) is the most frequently applied palliative treatment in HCC patients who are considered to be unsuitable for surgery and ablation. TACE involves the infusion of anticancer agents mixed with Lipiodol followed by the transient occlusion of tumor vessels with a gelatin sponge. However, TACE does not always result in complete necrosis of the tumor, and it is thought that patients who require TACE more than three times have difficulty in achieving complete remission.

Reports have previously shown that tumor angiogenesis is important for tumor growth and is regulated by angiogenic factors in many cancers including HCC (2). In particular, many reports have shown that the vascular endothelial growth factor (VEGF) is one of the most potent factors. In HCC, VEGF expression in tumor tissue has been reported to be correlated with aggressive behavior, early metastasis spread and poor prognosis (3-9). On the other hand, TACE promotes the development of angiogenesis in residual tumors through the up-regulation of VEGF expression, possibly due to the hypoxic insult (10). Therefore, it is hoped that some kind of adjuvant therapy after TACE, including one with an antiangiogenic function, would be more effective for treating HCC.

Prospective randomized studies have demonstrated that the postoperative administration of tegafur/uracil (UFT ${ }^{\circledR}$ ), an oral 5-fluorouracil (5-FU) derivative drug, can improve survival in lung, breast and colorectal cancer (11-14). UFT is an anti-tumor agent composed of tegafur [1-(2-tetrahydrofuryl)5-fluorouracil, FT] and uracil (U) at a molar ratio of 1:4. FT is a pro-drug that persistently releases 5-FU, and uracil is added to inhibit degradation of the released 5-FU. Yonekura et al demonstrated that UFT and its metabolites, $\gamma$-hydroxybutyric acid (GHB) and $\gamma$-butyrolactone (GBL), inhibited tumor angiogenesis (15). In the present study, we assessed whether UFT administration after treatment with TACE is efficacious as an adjuvant and anti-tumor angiogenesis treatment in patients with HCC. 


\section{Patients and methods}

Patient eligibility. Eligibility criteria were: HCC not suitable for surgical resection, liver transplantation or percutaneous ablation and treatment with TACE alone more than three times followed by recurrence within 6 months. The diagnosis of HCC was based on the results of radiological investigations [ultrasound, computed tomography (CT), magnetic resonance imaging (MRI), hepatic angiography] together with elevated levels of tumor markers [ $\alpha$-fetoprotein (AFP)] and protein induced by the vitamin $\mathrm{K}$ antagonist-II (PIVKA-II). The exclusion criteria were: age $<18$ years, serum creatinine $>1.5 \mathrm{mg} / \mathrm{dl}$, serum bilirubin $>3.0 \mathrm{mg} / \mathrm{dl}$, a Child-Pugh class $\mathrm{C}$, a WHO performance status $>2$, presenting major portal vein thrombosis, extrahepatic metastases, hematopoietic function with a platelet count of $<70.000 / \mathrm{mm}^{3}$ and a leukocyte count of $<3.000 / \mathrm{mm}^{3}$, and prior treatment with UFT. Between October 2003 and December 2005, 30 eligible patients were enrolled. The patients were randomly assigned to the UFT and control groups after TACE. As shown in Tables I and II, the patients and tumor characteristics were well balanced between the two groups. The mean age was 67.4 [standard deviation (SD), 0.4 years]. Most patients were men with underlying cirrhosis based on HCV. In both arms, cytological or histological tumor diagnosis was performed in a majority of patients.

Study design. All the patients provided informed consent. Since a placebo was unavailable, the study was not blinded. TACE was performed. Briefly, the catheter tip was advanced as near as possible to the site of the feeding artery. The emulsion of anticancer agents, mitomycin $\mathrm{C}$ and epirubicin and lipiodol followed by gelatin sponge particles was carefully injected under X-ray monitoring. The doses of emulsion and particles were determined based on the tumor size and extension of the lesions. Patient eligibility by means of examination by interview and a laboratory examination were confirmed at 3 days after TACE and the patients were then randomly assigned to the UFT and control groups. The control group was arranged in order to minimize bias in this study, and received TACE alone. In the UFT group, $300 \mathrm{mg}$ of UFT (Taiho Pharmaceutical Co., Tokyo, Japan) was administered daily. After the treatment, patients in the two groups underwent an ultrasonography and a measurement of the tumor markers (AFP and PIVKA-II) every month, and a dynamic computed tomography (CT) scan at 1 and 6 months after TACE. If intrahepatic recurrence was suspected, a dynamic CT scan was performed immediately. In patients who had recurrence or serious adverse events, treatment with UFT was withdrawn. Otherwise, observation and administration were continued up to 6 months after TACE. The serum VEGF level was determined to compare the fluctuations in angiogenesis between the two groups. Peripheral blood samples were taken from all patients at $0,1,3,7$ and 14 days after TACE and the serum VEGF level was measured with a VEGF immunoassay kit (R\&D Systems Inc., USA).

Evaluation of the response. The evaluation of TACE was based on the response evaluation criteria in solid tumors (RECIST) (16) and performed according to the recommendations of the Japanese Liver Cancer Study Group (17). Briefly, tumor
Table I. Response evaluation criteria.

\section{Treatment effect (TE)}

TE4: Necrosis rate $100 \%$ or reduction rate $100 \%$

TE3: Necrosis rate $50 \%$ and above, below $100 \%$

or

Reduction rate $50 \%$ and above, below $100 \%$

TE2: $\quad$ Exception of TE1 and TE3

TE1: $\quad$ Tumor growth $25 \%$ and above

\begin{tabular}{lcc} 
Overall response & TE & Tumor marker \\
\hline CR & TE4 & Normalization \\
PR & TE3 & Descent \\
SD & TE2 & Flatten \\
PD & TE1 & Elevation \\
\hline
\end{tabular}

$\mathrm{CR}$, complete response; $\mathrm{PR}$, partial response; $\mathrm{SD}$, stable disease and $\mathrm{PD}$, progressed disease.

necrosis and the reduction rate were classified into four degrees: treatment effect (TE) 1-4 according to the enhancement effect by a dynamic CT scan (Table I). Overall response was defined according to the tumor markers and the degree of TE at six months after TACE (Table I). The tumor necrosis rate was judged independently by at least one hepatologist and one radiologist. Recurrence was defined as lesions with typical findings of HCC on the imaging methods.

Assessment of the end points. The primary end point was the time to treatment failure (TTF) after TACE. The secondary end points were overall response rate and toxicity. The adverse events were based on the common terminology criteria for adverse events (CTCAE) v3.0. All categories of toxicity and complications of the treatment were recorded. Fluctuations of the serum VEGF were also evaluated as were other study variables.

Statistical considerations. This was a single-institution, openlabel, randomized phase II study that was conducted to generate pilot data regarding the preliminary efficacy and toxicity of UFT in combination with TACE. The planned sample size of 28 patients was calculated with a two-tailed type I error of $5 \%$ and a statistical power of $80 \%$, and the following hypotheses: an expected recurrence-free rate of 6 months would be $<1 \%$ in the control group and $30 \%$ in the UFT group. The primary end point was TTF, as previously defined. The TTF was generated using the Kaplan-Meier method, along with logrank tests for the differences. In order to estimate the significance of the UFT arm in the prolongation of TTF, multivariate analyses were performed using the Cox proportional hazard model. The following variables were considered for their effective value: tumor size and multiplicity, TNM classification, the number of TACE sessions, UFT administration, serum albumin and serum bilirubin levels, 
Table II. Baseline patient characteristics.

\begin{tabular}{|c|c|c|c|c|c|c|}
\hline & \multicolumn{3}{|c|}{ UFT group (n=14) } & \multicolumn{3}{|c|}{ Control group $(n=14)$} \\
\hline & & tients & $\%$ & & No. of patients & $\%$ \\
\hline \multicolumn{7}{|l|}{ Age (years) } \\
\hline Median & & 71 & & & 65 & \\
\hline Range & & $62-78$ & & & $55-79$ & \\
\hline \multicolumn{7}{|l|}{ Gender } \\
\hline Male & 12 & & 85.7 & 10 & & 71.4 \\
\hline Female & 2 & & 14.3 & 4 & & 28.6 \\
\hline Underlying cirrhosis & & 14 & & & 14 & \\
\hline \multicolumn{7}{|l|}{ Etiology of cirrhosis } \\
\hline Hepatitis C virus & 12 & & 85.8 & 13 & & 92.9 \\
\hline Hepatitis B virus & 1 & & 7.1 & 1 & & 7.1 \\
\hline Alcohol & 1 & & 7.1 & 0 & & 0.0 \\
\hline \multicolumn{7}{|l|}{ Child-Pugh class } \\
\hline A & 6 & & 42.9 & 8 & & 57.1 \\
\hline B & 8 & & 57.1 & 6 & & 42.9 \\
\hline \multicolumn{7}{|c|}{ ECOG performance status } \\
\hline 0 & 9 & & 64.3 & 9 & & 64.5 \\
\hline 1 & 5 & & 35.7 & 5 & & 35.7 \\
\hline 2 & 0 & & 0.0 & 0 & & 0.0 \\
\hline \multicolumn{7}{|c|}{ Serum bilirubin $(\mathrm{mg} / \mathrm{dl})$} \\
\hline Median & & 1.05 & & & 1.05 & \\
\hline Range & & $0.6-2.1$ & & & $0.4-1.8$ & \\
\hline \multicolumn{7}{|l|}{ Serum albumin $(\mathrm{g} / \mathrm{dl})$} \\
\hline Median & & 3.6 & & & 3.4 & \\
\hline Range & & $2.7-4.2$ & & & $2.4-4.1$ & \\
\hline \multicolumn{7}{|l|}{ Prothrombin time $(\%)$} \\
\hline Median & & 77.7 & & & 74.4 & \\
\hline Range & & $46.8-98.3$ & & & $59.6-117$ & \\
\hline \multicolumn{7}{|l|}{ Serum ALT (IU/l) } \\
\hline Median & & 48 & & & 48 & \\
\hline Range & & $28-101$ & & & $19-98$ & \\
\hline \multicolumn{7}{|c|}{ Platelet count $\left(\times 10^{3} / \mathrm{ml}\right)$} \\
\hline Median & & 8.2 & & & 7.9 & \\
\hline Range & & $5.2-14.1$ & & & $5.1-16.2$ & \\
\hline
\end{tabular}

ALT, alanine transaminase.

prothrombin time (PT), alanine transaminase (ALT), AFP and PIVKA-II. Data analysis was performed using the computer program StatView v5.0. The statistical difference was evaluated as significant at $\mathrm{P}<0.05$ in all analyses.

\section{Results}

Characteristics of patients and observation. Thirty patients were randomized into the study. Only two patients were excluded for the reasons shown in Fig. 1. The remaining 28 patients were randomly assigned to either the UFT $(n=14)$ or the control group $(n=14)$. All of the 28 patients were eligible for the response and toxicity analysis. The administration of UFT was discontinued in only one patient, for gastrointestinal symptoms. Table II outlines the demographic and clinical characteristics of the two groups. The median age was 65 in the control group and 71 years in the UFT group. The male to female ratios were 10:4 in the control group and 12:2 in the UFT group. All the 28 patients had an ambulatory performance status and $64.3 \%(18 / 28)$ were excellent 


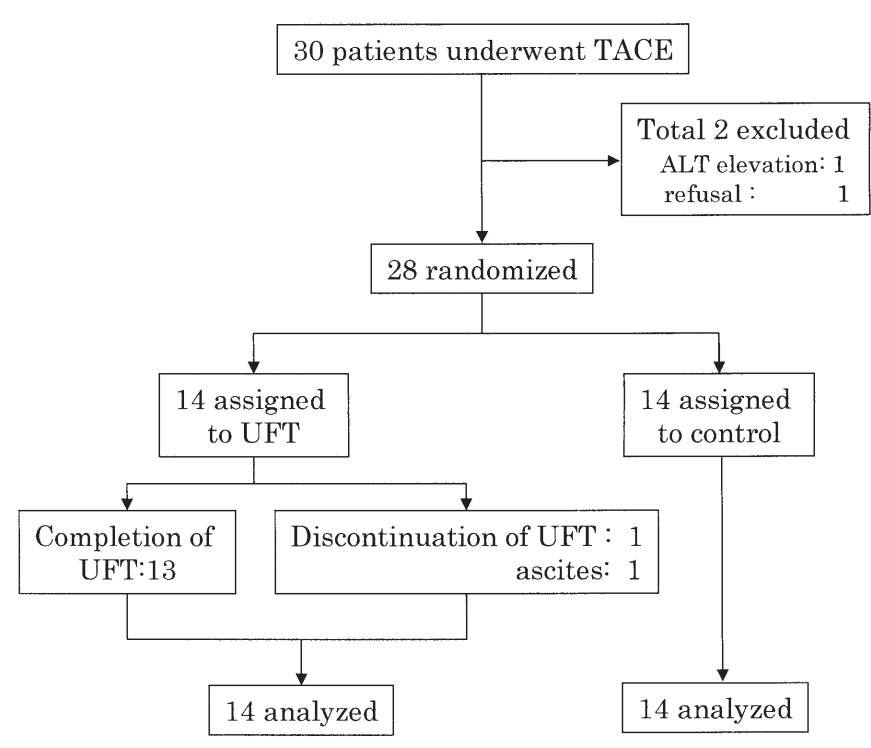

Figure 1. The trial profile. TACE, transcatheter arterial chemoembolization; UFT, tegafur/uracil; ALT, alanine transaminase.

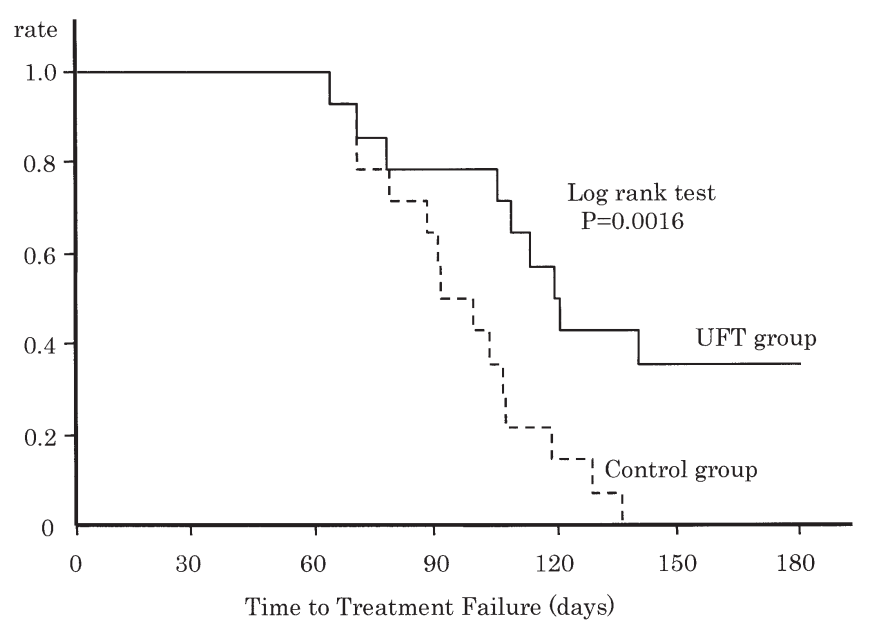

Figure 2. Time to treatment failure (TTF) curves of the UFT group (solid line) and the control group (dotted line). The median TTF in the UFT group was significantly prolonged as compared to that in the control group $(\mathrm{P}=0.0016)$. The overall response rate in the UFT group was also significantly higher than that in the control group.

(performance status 0). There was no statistically significant difference between the two groups with regard to age, gender, liver function, positivity of serum anti-HCV or hepatitis B surface antigen (HBsAg) and performance status. The groups were also balanced for the size and multiplicity of the tumors (Table III). There was no major imbalance in the number of TACE sessions between the two groups.

The tumor response rate and time to treatment failure (TTF) in the two groups. As for the primary endpoint, the median TTF in the control group and that in the UFT group were 87 and 127 days, respectively (Fig. 2). There was significant improvement $(\mathrm{P}=0.0016)$ in the time to recurrence for patients receiving adjuvant chemotherapy. Response data are

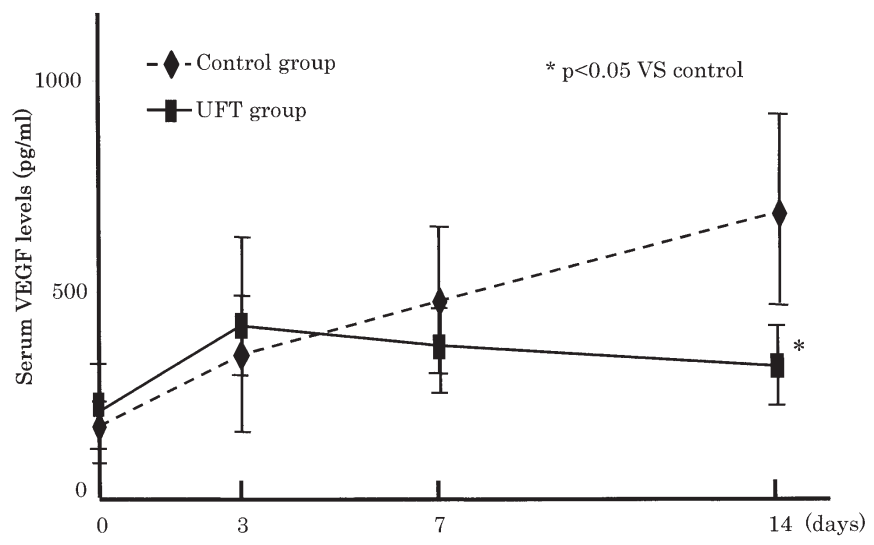

Figure 3. Mean serum vascular endothelial growth factor levels in the UFT group (solid line) and the control group (dotted line). Although the serum VEGF levels significantly increased in the control group, there was no significant increase in those levels in the UFT group at 7-14 days after the TACE. $\mathrm{P}<0.05$ compared with the values of the control group; bars, SD.

summarized in Table IV for all the patients. Of the 14 patients in the control group, all patients had responded to therapy TE4 in 13 and TE3 in 1) at one month as did all of the patients in the UFT group (TE4 in 14). The response data indicated similar response rates for the two groups and there was no statistical difference at one month after TACE. On the other hand, the overall response rates in the UFT and in the control groups were 35.7 and $0 \%$, respectively. There was statistical difference between the two groups. These data suggest that UFT administration had an additive advantage in the HCC patients with TACE.

The evaluation of adjuvant chemotherapy by multivariate analysis. Multivariate analysis was performed by using 12 factors considered to be important for the response to TACE. As shown in Table $\mathrm{V}$, the two factors of serum bilirubin level and UFT administration, were significantly associated with the response to TACE. In addition, the number of TACE sessions and baseline sum longest diameter influenced the response. These data indicate that UFT strongly contributed to improving response to TACE, and that a poorer liver function and more advanced HCC are risk factors contributing to recurrence, consistent with another report (18).

Regimen-related toxicity. With regard to toxicity, the adverse events observed in the UFT group were grade 2 ascites, grade 1 stomatitis, grade 1 diarrhea and grade 1 leucopenia (Table VI). The patient who had ascites was discontinued of UFT treatment and recovered promptly. Adverse events of more than grade 3 did not develop in any category. Serious complications by TACE such as liver failure were not observed in either group.

Fluctuation of the serum VEGF. As shown in Fig. 3, the serum VEGF levels were not different between the UFT and the control groups before TACE. Although the serum VEGF levels significantly increased in the control group, there was no significant increase of those levels in the UFT group at 7-14 days after embolization. 
Table III. Baseline tumor characteristics.

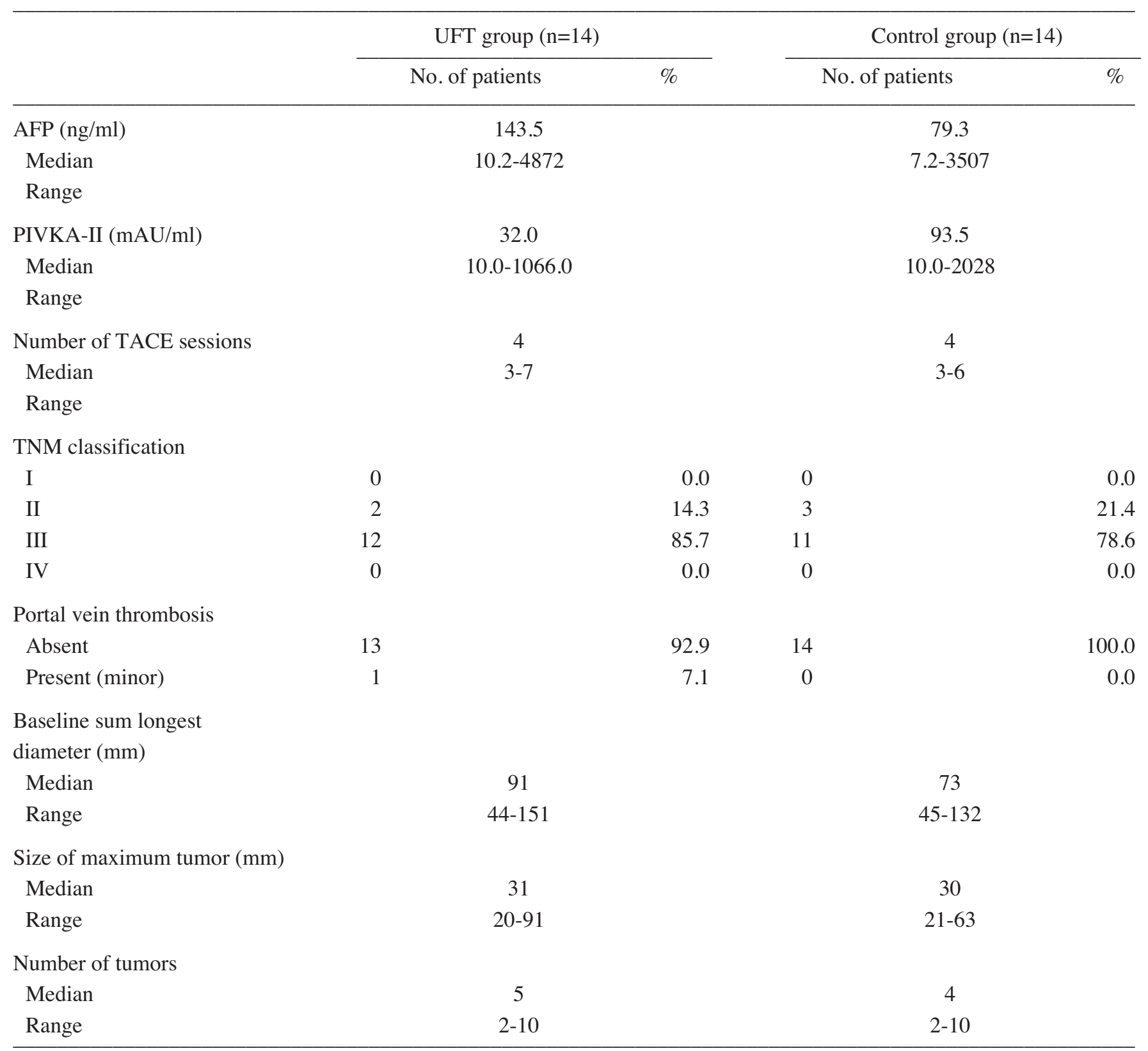

PIVKA-II, protein induced by vitamin K antagonist-II; AFP, $\alpha$-fetoprotein; TACE, transcatheter arterial chemoembolization.

Table IV. Response evaluation.

\begin{tabular}{|c|c|c|c|c|c|c|c|c|c|}
\hline & \multicolumn{4}{|c|}{ One month } & & \multicolumn{4}{|c|}{ Six months } \\
\hline & $\begin{array}{l}\text { UFT } \\
\text { No. }\end{array}$ & $\begin{array}{c}\text { group } \\
\%\end{array}$ & $\begin{array}{c}\text { Control } \\
\text { No. }\end{array}$ & $\begin{array}{c}\text { group } \\
\%\end{array}$ & & $\begin{array}{l}\text { UFT } \\
\text { No. }\end{array}$ & $\begin{array}{c}\text { group } \\
\%\end{array}$ & $\begin{array}{c}\text { Control } \\
\text { No. }\end{array}$ & $\begin{array}{c}\text { group } \\
\%\end{array}$ \\
\hline TE4 & 14 & 100 & 13 & 92.9 & $\mathrm{CR}$ & 1 & 7.1 & 0 & 0.0 \\
\hline TE3 & 0 & 0 & 1 & 7.1 & PR & 4 & 28.6 & 0 & 0.0 \\
\hline TE3+TE4 & 14 & 100 & 14 & 100.0 & $\mathrm{CR}+\mathrm{PR}$ & 5 & $35.7^{\mathrm{a}}$ & 0 & 0.0 \\
\hline TE2 & 0 & 0 & 0 & 0.0 & $\mathrm{NC}$ & 0 & 0 & 1 & 7.1 \\
\hline TE1 & 0 & 0 & 0 & 0.0 & PD & 9 & 64.3 & 13 & 92.9 \\
\hline
\end{tabular}

CR, complete response; $\mathrm{PR}$, partial response; $\mathrm{SD}$, stable disease; $\mathrm{PD}$, progressed disease. ${ }^{\mathrm{a}} \mathrm{P}<0.05$ vs control. 
Table V. A multivariate analysis of factors affecting the response duration using the Cox proportion hazard model.

\begin{tabular}{lcc}
\hline Variables & \multicolumn{1}{c}{$\begin{array}{c}\text { Hazard ratio } \\
(95 \% \mathrm{CI})\end{array}$} & P-value \\
\hline Albumin & $0.311(0.032-3.013)$ & 0.3132 \\
Bilirubin & $0.151(0.030-0.763)$ & $0.0222^{\mathrm{a}}$ \\
PT\% & $0.967(0.894-1.046)$ & 0.4026 \\
ALT & $1.017(0.981-1.055)$ & 0.3606 \\
AFP & $1.000(1.000-1.001)$ & 0.5496 \\
PIVKA-II & $1.000(0.998-1.002)$ & 0.8648 \\
TNM classification & $0.988(0.095-10.262)$ & 0.9917 \\
UFT administration & $6.630(1.733-24.369)$ & $0.0057^{\mathrm{b}}$ \\
Number of tumors & $0.872(0.590-1.289)$ & 0.4934 \\
Number of TACE & $1.722(0.913-3.247)$ & 0.0930 \\
sessions & & \\
Baseline sum longest & $1.552(0.926-2.603)$ & 0.0952 \\
diameter (mm) & & \\
Size of maximum & & 0.2065 \\
tumor (mm) & $0.729(0.447-1.190)$ & \\
\hline
\end{tabular}

\section{Discussion}

TACE has been widely used for the palliative treatment of HCC that is not suitable for curative therapy. Randomized controlled trials and prospective studies indicated that TACE is effective for improving the survival of patients with unresectable HCC (19-21). However, TACE alone does not always achieve complete necrosis of the tumor (22), and repetition of the treatment has been recommended for this modality of therapy (23). As is often the case with advanced HCC, such as in our subjects, frequent recurrences are caused by tumor angiogenesis and incomplete embolism. Therefore, another therapeutic approach, including chemotherapy or antiangiogenic treatment, has been expected to improve the response to TACE. Clinical trials using molecular-targeted agents, such as Bevacizumab or Sorafenib, have been started. However, these agents are unavailable at present. Retinoids (24) and Interferon $(25,26)$ have also been used as an adjuvant therapy to prevent the development of tumors, but the value of these treatments is not widely accepted in spite of recent promising results. UFT has been reported to be effective against HCC (27-29) and to have an antiangiogenic function $(15,30)$. We therefore expected that it would prevent recurrence after embolization.

As expected, our data demonstrated that UFT administration significantly improved TTF and the overall response rate compared to the control, and that UFT administration was an independently important factor that improved the response to TACE (Table V). In contrast to our result, Hasegawa et al recently reported that there was no evidence to support the potential benefits of adjuvant chemotherapy with UFT after surgery in patients with HCC (31). These apparently paradoxical results may be attributed to the difference in the target. In that report, UFT was expected to
Table VI. Common terminology criteria for adverse events, (CTCAE) v3.0 in the UFT group.

\begin{tabular}{lccccc}
\hline & & \multicolumn{4}{c}{ Grade } \\
\cline { 3 - 6 } & 1 & 2 & $3-4$ & Overall \\
Adverse event & $($ No. $)$ & $($ No. $)$ & $($ No. $)$ & No. & $\%$ \\
\hline Blood/bone marrow & & & & & \\
$\quad$ Leukocytes & 1 & - & - & 1 & 7.1 \\
Gastrointestinal & & & & & \\
$\quad$ Stomatitis - oral cavity & 1 & - & - & 1 & 7.1 \\
$\quad$ Ascites (non-malignant) & - & 1 & - & 1 & 7.1 \\
Diarrhea & 1 & - & - & 1 & 7.1 \\
\hline
\end{tabular}

prevent metastatic recurrence caused by HCC cells present in the microcirculation. In our study, the expected target was the chemotherapeutic effect against viable HCC and antiangiogenesis after TACE. Therefore, the findings of the present study do not necessarily conflict with the above report and support that UFT may be a useful adjunct.

However, it is also a fact that the majority of patients still had recurrence in our study. The reasons for the recurrence in the UFT group may be the small dosage of UFT and tolerance to UFT. All adverse events present in the UFT group were fortunately mild to moderate (Table VI), and it may be worthwhile to administer higher doses of UFT. However, this may cause serious adverse effects on liver function because almost all of the patients with HCC also have cirrhosis and pancytopenia derived from hypersplenism. A previous report showed that the appearance rate of ascites or encephalopathy was significantly high and bilirubin also became significantly high (32). Alternatively, an attempt may be made to administer other 5-FU related agents including S-1 and capecitabine. From the viewpoint of the inhibition of angiogenesis due to VEGF, it is reasonable to administer UFT at three days after TACE because the gelatin sponge dissolves and disappears within a few weeks $(33,34)$ and it has been reported that the VEGF level increases at 7-14 days after TACE $(10,35)$. Meticulous attention to adverse events is essential when employing chemotherapy, even as an adjuvant therapy.

Notably, our data indicated that increases in the serum VEGF levels after TACE were inhibited in the UFT group, which may have been secondary to the chemotherapeutic effect because previous reports $(15,30)$ have shown that the metabolites of UFT inhibit the VEGF function but not the production of VEGF, although the possibility remains that the metabolites of UFT inhibit VEGF production under a hypoxic condition. These data suggest that this adjuvant therapy may have primary and secondary antiangiogenic effects.

In conclusion, our study indicated that the UFT treatment was advantageous in terms of TTF and the response rate to TACE, although the number of patients studied was not very large. If any combined regimens are attempted for HCC patients with TACE in order to prolong the response, adjuvant chemotherapy should include angiogenesis inhibitors as well as UFT because of the hypervascular nature of HCC tumors. 


\section{References}

1. Parkin DM, Bray F, Ferlay J and Pisani P: Estimating the world cancer burden: Globocan 2000. Int J Cancer 94: 153-156, 2001.

2. El-Assal ON, Yamanoi A, Soda Y, et al: Clinical significance of microvessel density and vascular endothelial growth factor expression in hepatocellular carcinoma and surrounding liver: possible involvement of vascular endothelial growth factor in the angiogenesis of cirrhotic liver. Hepatology 27: 1554-1562, 1998.

3. Miura H, Miyazaki T, Kuroda M, et al: Increased expression of vascular endothelial growth factor in human hepatocellular carcinoma. J Hepatol 27: 854-861, 1997.

4. Mise M, Arii S, Higashituji $\mathrm{H}$, et al: Clinical significance of vascular endothelial growth factor and basic fibroblast growth factor gene expression in liver tumor. Hepatology 23: 455-464, 1996.

5. Torimura $\mathrm{T}$, Sata $\mathrm{M}$, Ueno $\mathrm{T}$, et al: Increased expression of vascular endothelial growth factor is associated with tumor progression in hepatocellular carcinoma. Hum Pathol 29: 986-991, 1998.

6. Li XM, Tang ZY, Zhou G, Lui YK and Ye SL: Significance of vascular endothelial growth factor mRNA expression in invasion and metastasis of hepatocellular carcinoma. J Exp Clin Cancer Res 17: 13-17, 1998.

7. Yamaguchi R, Yano H, Iemura A, Ogasawa S, Haramaki M and Kojiro M: Expression of vascular endothelial growth factor in human hepatocellualr carcinoma. Hepatology 28: 68-77, 1998.

8. Poon RT, Lau CP, Ho JW, Yu WC, Fan ST and Wong J: Tissue factor expression correlates with tumor angiogenesis and invasiveness in human hepatocellular carcinoma. Clin Cancer Res 9: 5339-5345, 2003.

9. Schmitt M, Horbach A, Kubitz R, Frilling A and Haussinger D: Disruption of hepatocellular tight junctions by vascular endothelial growth factor (VEGF): a novel mechanism for tumor invasion. J Hepatol 41: 274-283, 2004.

10. Suzuki H, Mori M, Kawaguchi C, Adachi M, Miura S and Ishii H: Serum vascular endothelial growth factor in the course of transcatheter arterial embolization of hepatocellular carcinoma. Int J Oncol 14: 1087-1090, 1999.

11. Hamada C, Tanaka F, Ohta M, et al: Meta-analysis of postoperative adjuvant chemotherapy with Tegafur-Uracil in nonsmall-cell lung cancer. J Clin Oncol 23: 4999-5006, 2005.

12. Noguchi S, Koyama H, Uchino J, et al: Postoperative adjuvant therapy with tamoxifen, tegafur plus uracil, or both in women with node-negative breast cancer: a pooled analysis of six randomized controlled trials. J Clin Oncol 23: 2172-2184, 2005.

13. Sakamoto J, Hamada C, Yoshida S, et al: An individual patient data meta-analysis of adjuvant therapy with uracil-tegafur (UFT) in patients with curatively resected rectal cancer. Br J Cancer 96: 1170-1177, 2007.

14. Kato H, Ichinose Y, Ohta M, Hata E, Tsubota N, Tada H, et al: A randomized trial of adjuvant chemotherapy with uracil-tegafur for adenocarcinoma of the lung. N Engl J Med 350: 1713-1721, 2004.

15. Yonekura K, Basaki Y, Chikahisa L, et al: UFT and its metabolites inhibit the angiogenesis induced by murine renal cell carcinoma, as determined by a dorsal air sac assay in mice. Clin Cancer Res 5: 2185-2191, 1999.

16. Therasse P, Arbuck SG, Eisenhauer EA , et al: New guidelines to evaluate the response to treatment in solid tumors. J Natl Cancer Inst 92: 205-216, 2000.

17. Japanese Liver Cancer Study Group of Japan: Criteria for the evaluation of direct effects of hepatocellular carcinoma. ACTA Hepatol Jpn 45: 380-385, 2004.

18. Arimura E, Kotoh K, Nakamuta M, Morizono S, Enjoji M and Nawata $\mathrm{H}$ : Local recurrence is an important prognostic factor of hepatocellular carcinoma. World J Gastroenterol 11: 5601-5606, 2005.
19. Takayasu K, Arii S, Ikai I, et al: Liver Cancer Study Group of Japan. Prospective cohort study of transarterial chemoembolization for unresectable hepatocellular carcinoma in 8510 patients. Gastroenterology 131: 461-469, 2006.

20. Llovet JM, Real MI, Montana X, et al: Arterial embolisation or chemoembolisation versus symptomatic treatment in patients with unresectable hepatocellular carcinoma: a randomized controlled trial. Lancet 359: 1734-1739, 2002.

21. Lo CM, Ngan H, Tso WK, Liu CL, Lam CM, Poon RT, Fan ST and Wong J: Randomized controlled trial of transarterial lipiodol chemoembolization for unresectable hepatocellular carcinoma. Hepatology 35: 1164-1171, 2002.

22. Wakasa K, Sakurai M, Kuroda C, Marukawa T, Monden M, Okamura $\mathrm{J}$ and Kurata A: Effect of transcatheter arterial embolization on the boundary architecture of hepatocellular carcinoma. Cancer 65: 913-919, 1990.

23. Ikeda K, Kumada H, Saitoh S, Arase Y and Chayama K: Effect of repeated transcatheter arterial embolization on the survival time in patients with hepatocellular carcinoma. Cancer 68: 2150-2154, 1991

24. Muto Y, Moriwaki H, Ninomiya M, et al: Prevention of second primary tumors by an acycric retinoid, polyprenoic acid, in patients with hepatocellular carcinoma. N Eng J Med 334: 1561-1567, 1996.

25. Kubo S, Nishiguchi S, Hirohashi K, et al: Effects of long-term postoperative interferon-alpha therapy on intrahepatic recurrence after resection of hepatitis $\mathrm{C}$ virus-related hepatocellular carcinoma. A randomized, controlled trial. Ann Intern Med 134: 963-967, 2001.

26. Shiratori Y, Shiina S, Teratani T, et al: Interferon therapy after tumor ablation improves prognosis in patients with hepatocellular carcinoma associated with hepatitis $C$ virus. Ann Intern Med 138: 299-306, 2003.

27. Yamamoto M, Arii S, Sugahara K and Tobe T: Adjuvant oral chemotherapy to prevent recurrence after curative resection for hepatocellular carcinoma. Br J Surg 83: 336-340, 1996.

28. Ishikawa $\mathrm{T}$, Ichida $\mathrm{T}$, Ishimoto $\mathrm{Y}$, et al: Complete remission of multiple hepatocellular carcinomas associated with hepatitis $\mathrm{C}$ virus-related, decompensated liver cirrhosis by oral administration of enteric-coated tegafur/uracil. Am J Gastroenterol 94: 1682-1685, 1999.

29. Ishikawa T, Ichida T, Sugitani S, et al: Improved survival with oral administration of enteric-coated tegafur/uracil for advanced stage IV - A hepatocellular carcinoma. J Gastroenterol Hepatol 16: 452-459, 2001.

30. Basaki Y, Chikahisa L, Aoyagi K, et al: Gamma-Hydroxybutyric acid and 5-fluorouracil, metabolites of UFT, inhibit the angiogenesis induced by vascular endothelial growth factor. Angiogenesis 4: 163-173, 2001.

31. Hasegawa K, Takayama T, Ijichi M, et al: Uracil-tegafur as an adjuvant for hepatocellular carcinoma: a randomized trial. Hepatology 44: 891-895, 2006.

32. Ikeda K, Saitoh S, Koida I, Tsubota A, Arase Y, Chayama K and Kumada HA: A prospective randomized evaluation of a compound of tegafur and uracil as an adjuvant chemotherapy for hepatocellular carcinoma treated with transcatheter arterial chemoembolization. Am J Clin Oncol 18: 204-210, 1995.

33. Doyon DMA, Jourde AM, Regensberg C and Frileux C: L'embolisation arterielle hepatique dans les tumeurs malignes du foie. Ann Radiol 17: 593-603, 1974.

34. Yamada R, Nakatsuka H, Nakamura K, et al: Transcatheter arterial embolization therapy in unresectable hepatomas experience in 15 cases. ACTA Hepatol Jpn 20: 595-603, 1979

35. Liao XF, Yi JL, Li XR, Deng W, Yang ZF and Tian G: Angiogenesis in rabbit hepatic tumor after transcatheter arterial embolization. World J Gastroenterol 10: 1885-1889, 2004. 